\title{
Impact of phonon nonlocality on nanogap and nanolayer polar resonators
}

\author{
Christopher R. Gubbin and Simone De Liberato* \\ School of Physics and Astronomy, University of Southampton, Southampton, SO17 1BJ, United Kingdom
}

\begin{abstract}
Polar dielectric nanoresonators can support hybrid photon-phonon modes termed surface phonon polaritons with lengthscales below the diffraction limit. In the deep sub-wavelength regime the optical response of these systems was shown to diverge from that predicted through a standard dielectric description. Recently we developed an analytical, dielectric approach and applied it to spheres and planar heterostructures, reproducing anomalous features observed in experiment and microscopic calculations. In this Letter we develop tools to describe the nonlocal response of polar nanoresonators of arbitrary symmetry, and use them to investigate systems with nanogaps and nanolayers of practical technological relevance. We demonstrate that the available field enhancement is strongly reduced, as the electromagnetic energy leaks away from the hotspots, while phononic resonances are shifted by resonator effects.
\end{abstract}

Nanophotonics is concerned with concentration and control of light on deep-subwavelength scales. This is possible by exploiting kinetic motion of charged particles, allowing the diffraction limit to be beaten many times over $^{1}$. This is the basis for polar nanophotonics, where photons are hybridised with the optic phonons of a crystal lattice in modes termed surface phonon polaritons ${ }^{2-4}$. These modes are highly tuneable ${ }^{5-10}$ and have broad applications in nonlinear optics ${ }^{11,12}$ and the fabrication of nanophotonic circuitry ${ }^{13-15}$.

A key benefit of localised surface phonon resonances is their strong morphological dependance. In geometries containing sharp corners or small gaps this results in a dramatic increase in local energy density which can be used for sensing applications ${ }^{16}$. When confinement length approaches the atomic length scale the finite wavelength of the longitudinal optic (LO) and transverse optic (TO) phonons become important. Propagative LO modes affect screening charges induced at the particle boundary. This is not accounted for in local theories of dielectric response, which assume screening charges are exactly localised at the scatterer boundary. The nonlocal regime has been studied in plasmonic systems, where excitation of strongly evanescent bulk plasma waves smear charge, limiting maximal field enhancement and blue shifting modal frequencies ${ }^{17,18}$.

The nonlocal regime is difficult to access, requiring fabrication of nanoscale resonators or gaps ${ }^{19}$. It is however expected to be of particular interest for phonon polaritons in the field of crystal hybrids, which are constructed from many alternating nanoscale layers of different polar dielectric materials. In these systems recent studies have shown strong divergence from the local optical response ${ }^{20}$. As nonlocality implies a transfer of energy from the electromagnetic field to elastic deformation of the lattice, understanding the nonlocal physics is also necessary to assess the suitability of these systems for field-enhancement-based applications such as surface-enhanced infrared absorption spectroscopy, or single-molecule strong coupling ${ }^{21,22}$. Nonlocal effects can be modelled using a first-principles method such as density functional theory, ${ }^{23,24}$ which however scales badly to realistic devices. To provide more agile approaches we recently developed an analytical continuum theory, describing polar nonlocality in terms of macroscopic fields. The validity of this model was confirmed by comparison to recent experiments for structures with features below $2 \mathrm{~nm}$ in $\operatorname{size}^{20,25}$. Such analytical approaches are tractable in systems with strong symmetry but cannot be easily generalised.

In this Letter we develop numerical tools to describe the nonlocal response of polar nanosystems with arbitrary geometry and use them to investigate the nonlocal phenomenology of technologically relevant nano-objects. This is achieved through integration of our nonlocal response theory with COMSOL Multiphysics, a commercial finite element solver, the model is distributed for the use of the community ${ }^{26}$. The only inputs parameters required by the model, beyond those required for a local dielectric description, are the low-wavevector LO and TO phonon velocities which can be parameterised utilising the bulk phonon dispersions, readily available in the literature for most materials. The numerical approach is validated by comparison to our previous analytical nonlocal scattering spectra for $3 \mathrm{C}-\mathrm{SiC}$ spheres ${ }^{25}$. The model is then applied to the study of spherical dimers, investigating the effect of nonlocality on field confinement. Finally we study the nonlocal response of macroscopic resonators containing nanoscale layers, showing the effect of nonlocality in nanostructured crystal hybrid resonators.

Our polar crystal is treated in the continuum limit as an isotropic lattice with a single phonon branch characterised by zone-centre LO (TO) phonon frequencies $\omega_{\mathrm{L}}\left(\omega_{\mathrm{T}}\right)$ in a quadratic dispersion approximation analogous to that used in nonlocal plasmonics ${ }^{17}$. Phonons couple to driving electric field $\mathbf{E}$ as

$$
\left[\omega_{\mathrm{T}}^{2}-\omega(\omega+i \gamma)\right] \mathbf{X}+\nabla \cdot \bar{\tau}-\frac{\mu}{\rho} \mathbf{E}=0
$$

in which $\mathbf{X}$ is the relative ionic displacement, $\gamma$ is the damping rate and $\rho$ and $\mu$ are effective mass and charge densities. The matrix $\bar{\tau}$ describes the phonon dispersion, acting as an effective stress tensor ${ }^{27}$ given for an isotropic lattice by

$$
\bar{\tau}=\beta_{\mathrm{T}}^{2}\left(\nabla \mathbf{X}+(\nabla \mathbf{X})^{\mathrm{T}}\right)+\left(\beta_{\mathrm{L}}^{2}-2 \beta_{\mathrm{T}}^{2}\right) \nabla \cdot \mathbf{X} \overline{\mathrm{I}}
$$


where $\beta_{\mathrm{T}}\left(\beta_{\mathrm{L}}\right)$ are phenomenological velocities describing TO (LO) phonon dispersion and $\overline{\mathrm{I}}$ the identity tensor. The model is completed by the constitutive relation

$$
\mathbf{P}=\mu \mathbf{X}+\epsilon_{0}\left(\epsilon_{\infty}-1\right) \mathbf{E},
$$

where $\epsilon_{\infty}$ is the high-frequency permittivity and the material polarisation is $\mathbf{P}$.

In a previous publication ${ }^{25}$ we solved Eq. 1 and Maxwell's equations analytically in simple systems of high symmetry: a sphere in vacuum and a suspended nanolayer. Here we employ a numerical method which permits easy nonuniform meshing as oscillations induced by Eq. 1 occur on the nanometer scale, while the wavelength of midinfrared photons is typically 4 orders of magnitude larger. Non-uniform meshing is simple using commercial finite element (FEM) solvers. To use Eq. 1 in a FEM calculation it must be translated into weak form. The strong statement is that the left-hand side of Eq. 1 is zero everywhere. The corresponding weak statement is that it, integrated over the computational domain and multiplied by a family of test functions $\boldsymbol{\Phi}$, is zero. Integrating by parts over the computational domain yields

$$
\begin{aligned}
\int \mathrm{d}^{3} \mathrm{r}[ & \frac{\mu}{\omega_{\mathrm{T}}^{2}-\omega(\omega+i \gamma)} \\
& \times\left(\beta_{\mathrm{L}}^{2}(\nabla \cdot \mathbf{X})(\nabla \cdot \mathbf{\Phi})-\beta_{\mathrm{T}}^{2}(\nabla \times \mathbf{X}) \cdot(\nabla \times \mathbf{\Phi})\right) \\
& \left.+\alpha \mathbf{X} \cdot \mathbf{\Phi}+\epsilon_{0}\left(\epsilon_{\mathrm{LRA}}(\omega)-\epsilon_{\infty}\right) \mathbf{E} \cdot \mathbf{\Phi}\right]=0
\end{aligned}
$$

where we simplified using the local dielectric function

$$
\epsilon_{\mathrm{LRA}}(\omega)=\epsilon_{\infty}\left[1+\frac{\alpha^{2} / \epsilon_{0} \epsilon_{\infty} \rho}{\omega_{\mathrm{T}}^{2}-\omega(\omega+i \gamma)}\right]
$$

In coupling Eq. 4 with Maxwell's equations we introduce the macroscopic fields, $\mathbf{X}$ and $\bar{\tau}$. This means the Maxwell boundary conditions are insufficient to determine mode amplitudes in each layer and additional boundary conditions $(\mathrm{ABC})$ are required. We provided an exhaustive discussion of the appropriate $\mathrm{ABC}$ to be used in polar dielectrics in Ref. ${ }^{25}$, to which the interested reader is invited to refer. For completeness in the following we provide a brief overview of this important problem. Considering energy transport across a material interface ${ }^{28}$, it is possible to derive the $\mathrm{ABC}$ to be satisfied at the interface between two nonlocal media: the continuity of the normal and in-plane ionic displacement $\mathbf{X}$ and of the normal and shear components of the effective stress $\bar{\tau} \cdot \hat{\mathbf{n}}$. At interfaces between local and nonlocal layers the appropriate combination of boundary conditions is actively debated $^{29-31}$. It is necessary to apply two conditions, leaving the remaining components discontinuous. We fix the normal component of the displacement, analogously to the plasmonic case ${ }^{17,18,32}$, and the normal component of the stress tensor, which provides the correct result in the case of vanishing $\beta_{\mathrm{T}}$.

To verify our model we study a system whose nonlo-

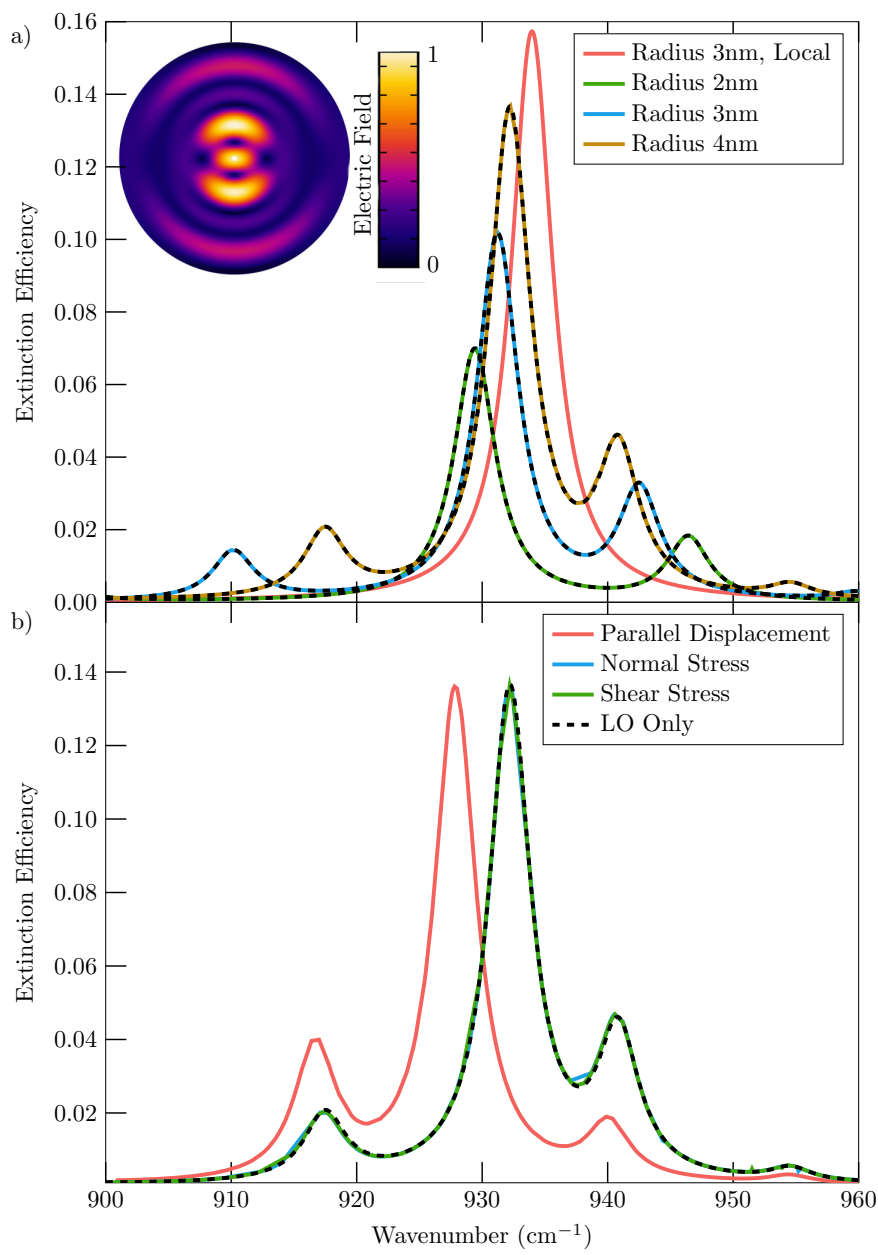

FIG. 1. a) Extinction efficiencies for spheres with $r=$ $4,3,2 \mathrm{~nm}$. Numerical data is shown by solid lines. Analytical data is shown by black dashed lines. The local result is also shown for $r=3 \mathrm{~nm}$. Inset shows nonlocal electric field for $r=4 \mathrm{~nm}$. b) Comparison of numerical extinction efficiencies using different ABC for $r=4 \mathrm{~nm}$.

cal response is analytically calculable. We demonstrated that nonlocal extinction spectra of nanoscopic $3 \mathrm{C}-\mathrm{SiC}$ spheres are well described by a quasistatic model in which the TO dispersion is neglected and only the boundary condition on the normal displacement is enforced ${ }^{25,32}$. This is a reasonable approximation near the Fröhlich resonance where the TO phonon is strongly evanescent. Considering the case $\beta_{\mathrm{L}}=15.39 \times 10^{5} \mathrm{~cm} \mathrm{~s}^{-133}$ we calculate nonlocal extinction efficiencies.

Results are shown in Fig. 1a for radius $r=2,3,4 \mathrm{~nm}$. In the local case small spheres exhibit a single resonance at the Fröhlich frequency, $\omega_{\mathrm{F}} \approx 933 \mathrm{~cm}^{-1}$, illustrated for $r=3 \mathrm{~nm}$ by the red curve. In the nonlocal case additional peaks appear in the extinction spectrum. These correspond to quantized LO phonon modes. Eventually the Fröhlich resonance red shifts as a result of an increase in the effective nonlocal dielectric function. Note that in Fig. 1a analytical (numerical) results are illus- 
trated by solid (dashed) lines, overlap is exact on this scale demonstrating the accuracy of our implementation. The inset shows the nonlocal electric field magnitude for the $4 \mathrm{~nm}$ sphere, the short wavelength LO phonon oscillation is clearly visible.

Eq. 1 is a continuum approximation, treating the phonon dispersion phenomenologically through effective stress tensor Eq. 2. Phonon dispersions are assumed quadratic, meaning LO and TO dispersion relations have solutions at all frequencies. In reality the granular structure of the lattice prevents this, resulting in a decrease in group velocity to zero at the Brillouin zone edge ${ }^{34}$. In $3 \mathrm{C}-\mathrm{SiC} \mathrm{TO}$ dispersion is weak, meaning that using finite $\beta_{\mathrm{T}}$ should not alter the extinction cross-section. We verify this in Fig. $1 \mathrm{~b}$ for a $r=4 \mathrm{~nm}$ and $\beta_{\mathrm{T}}=9.15 \times 10^{5} \mathrm{~cm} \mathrm{~s}^{-133}$, demonstrating that the $\mathrm{ABC}$ we chose overlaps with the $\beta_{\mathrm{T}} \approx 0 \mathrm{~cm} \mathrm{~s}^{-1}$ limit explored in the prior section. In the same panel we also plot results using different $\mathrm{ABC}$, showing how also fixing the shear stress provides essentially the same results, while using the in-plane displacement would lead to an unphysical redshift.

We have applied our numerical model to systems with analytical solutions, demonstrating its reliability. In the remainder of this Letter we apply it to nanophotonic systems relevant for technologically relevant surface phonon polaritonics ${ }^{6,35}$, where lack of symmetry prevents analytical solutions. Firstly we study the effect of nonlocality on field hotspots, predicted in plasmonic systems to result in strong charge smearing and a corresponding decrease in the maximal field ${ }^{36}$. We consider a spherical dimer, consisting of two $3 \mathrm{C}-\mathrm{SiC}$ spheres of radius $r=5 \mathrm{~nm}$, separated by a gap of width $d$. For large gaps the system modes are those of the isolated spheres studied in Fig. 1. For small $d$ these hybridise into bonding and antibonding resonances ${ }^{37}$, as shown in the local scattering spectra for $d=2 \mathrm{~nm}$ in Fig. $2 \mathrm{a}$ at $918 \mathrm{~cm}^{-1}$ and $935 \mathrm{~cm}^{-1}$ respectively. In the nonlocal spectra (solid line) these modes are supplemented by the LO modes supported by the dimer, as in Fig. 1a.

The antibonding mode is of most interest as opposing charges enclosing the gap result in strong capacitative field enhancement. This is demonstrated in the local case by the dashed line in Fig. 2a, which shows field enhancement at gap centre. On resonance in the local case this peaks at around 125 . In the nonlocal case the enhancement diminishes to around 40. This can be understood from the field intensity plots inset in Fig. 2b. In the local case field is strongly localised in the gap, and is efficiently screened from the sphere interior. In the nonlocal case screening is less efficient and induced screening charges smear into the spheres, diminishing capacitative charging of the dimer. Gap-dependance is demonstrated in Fig. 2b, where we plot peak field enhancement for the antibonding mode. In the local case field enhancement diverges as $d \rightarrow 0$, while in the nonlocal case this is offset by increased energy transfer to propagative LO modes in the nanosphere. Note that, as clear from the inset in Fig. 2b, longitudinal modes emit-
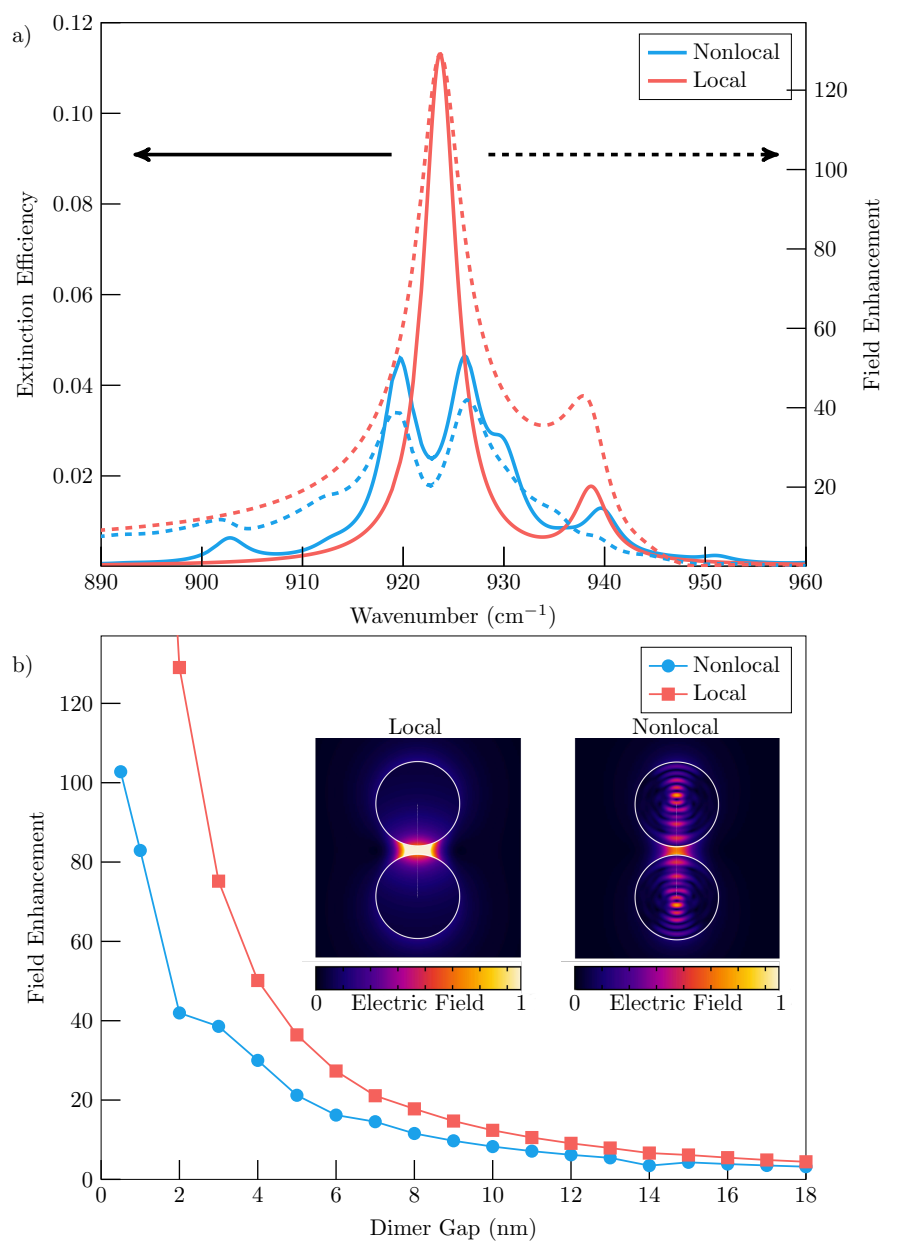

FIG. 2. a) Local (red) and nonlocal (blue) extinction efficiencies (solid) and field enhancements (dashed) at gap centre for a dimer of radius $r=5 \mathrm{~nm}$ and gap $d=2 \mathrm{~nm}$. b) Comparison of local (red squares) and nonlocal (blue circles) peak field enhancements for a dimer with $\mathrm{r}=5 \mathrm{~nm}$ as a function of gap width. Inset shows the electric field magnitude of the antibonding mode for $d=1 \mathrm{~nm}$.

ted in the nonlocal case form standing waves inside the nanospheres. The field can thus in principle be enhanced at its antinodes inside the dielectric. Although applications are normally predicated on enhancement exterior to the polar resonator ${ }^{21,22}$, we prefer to point out this feature as it stands in stark contrast to what happens in plasmonic systems, where the longitudinal modes are instead evanescent.

We have discussed nonlocality in systems of nanoscopic dimensions or with nanoscale air gaps. Fabrication of polar resonators on this scale is challenging, however making macroscopic heterostructures containing nanolayers is a well established process. It was recently suggested that in bulk polar superlattices, termed crystal hybrids ${ }^{20}$, a nonlocal description of the optical response is necessary ${ }^{25}$. Describing a system containing many polar layers is beyond the scope of this work, however we demonstrate the effect of nonlocality in larger resonators 


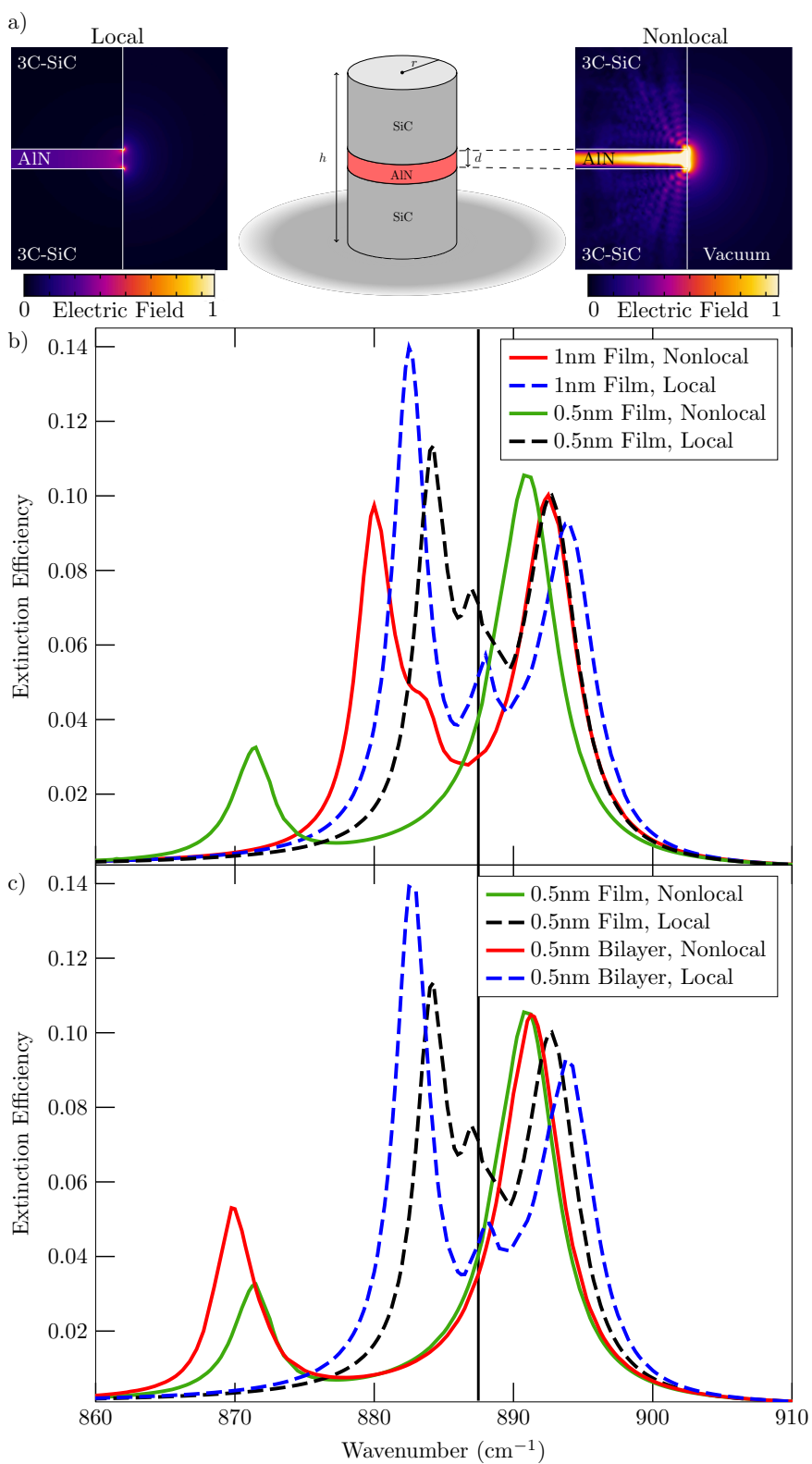

FIG. 3. a) Electric field intensity at the nanopillar boundary for local and nonlocal models, normalised to the maximal value in each case. b) Comparison of local (dashed) and nonlocal (solid) extinction efficiencies for a 3C-SiC nanopillar on substrate with $h=250 \mathrm{~nm}, 2 r=500 \mathrm{~nm}$ containing an AlN film of thickness 0.5 and $1 \mathrm{~nm}$. c) Comparison of local (dashed) and nonlocal (solid) extinction efficiencies for a 3CC$\mathrm{SiC}$ nanopillar on substrate with $h=250 \mathrm{~nm}, 2 r=500 \mathrm{~nm}$ containing one and two thin AlN films of thickness $0.5 \mathrm{~nm}$

containing a few nanoscopic polar layers.

We apply our model to a typical polar resonator, a 3C$\mathrm{SiC}$ nanopillar of height $h=250 \mathrm{~nm}$ and radius $r=$ $500 \mathrm{~nm}$ on same material substrate ${ }^{6,35}$. Considering a single AlN layer in the pillar centre as in Fig. 3a, with $\beta_{\mathrm{T}}=1 \times 10^{5} \mathrm{~cm} \mathrm{~s}^{-1}, \beta_{\mathrm{L}}=5.1 \times 10^{5} \mathrm{~cm} \mathrm{~s}^{-125}$. Dashed lines in Fig. 3b show the local extinction efficiency for film thicknesses $d=0.5,1 \mathrm{~nm}$. The spectrum shows three features. The first, closely resonant with the zone-centre LO phonon in the AlN $\left(\omega_{\mathrm{L}} \approx 887 \mathrm{~cm}^{-1}\right.$, marked by the vertical line) is the Berreman mode of the AlN film ${ }^{7}$, independent of the pillar dimensions. Other peaks are photonic modes resulting from hybridisation of the monopolar mode of the nanopillar ${ }^{6}$ with the epsilon-near-zero response of the $\mathrm{AlN}^{7}$. In the $1 \mathrm{~nm}$ case these are more strongly split around $\omega_{\mathrm{L}}$ as increased film thickness leads to enhanced pillar-film coupling. In the nonlocal case (solid lines in Fig. 3b) the Berreman mode red shifts and weakens as a result of the quantisation of LO phonon resonances in the film, and it is only visible as a shoulder around $882 \mathrm{~cm}^{-1}$ for the $1 \mathrm{~nm}$ film. In the local case all LO phonon modes sit at exactly $\omega_{\mathrm{L}}$, yielding a single resonance with large oscillator strength. In the nonlocal case phonons of differing out-of-plane wavevectors have different frequencies, smaller than $\omega_{\mathrm{L}}$, thus splitting the Berreman resonance into a set of discrete peaks each of which has diminished oscillator strength. Additionally photonic modes are red shifted, this is particularly true for the low frequency mode. The red shift is more pronounced for the $0.5 \mathrm{~nm}$ film. This is because, as demonstrated in Ref. ${ }^{25}$ for freestanding films, thin AlN films support quantised Fabry-Pérot LO phonon modes which red shift as $d \rightarrow 0$, resulting in a red shift of the hybridised resonances.

To demonstrate how these results of can be extrapolated to crystal hybrids comprising multiple crystal layers, we consider the effect of adding a second $0.5 \mathrm{~nm}$ AlN film, separated from the first by a $0.5 \mathrm{~nm} 3 \mathrm{C}-\mathrm{SiC}$ spacer layer, results are shown in Fig. 3c. In the local (dashed lines) cases the additional layers results in an increased splitting of the photonic modes around $\omega_{\mathrm{L}}$ as a result of increased coupling between the AlN epsilon-near-zero mode and the pillar resonance. In the nonlocal case the same effect is observable around the red-shifted fundamental Fabry-Pérot LO phonon resonance. Also shown in Fig. 3a are the electric field magnitude in the nanopillar for a $1 \mathrm{~nm}$ AlN film. In the local case the field is localised at the pillar edge ${ }^{9}$. In the nonlocal case propagative LO modes cause electromagnetic energy to leach into the pillar, smearing field hotspots.

We developed a numerical method to study the nonlocal response of nanoscopic polar resonators, and applied it to geometries of practical relevance for current nanophotonic investigations. We demonstrated that nonlocal effects can lead to a strong reduction in the achievable field enhancement in structures with nanoscopic features, allowing the electromagnetic energy to propagate in the bulk in the form of LO phonons.

We also studied the nonlocal phenomenology of cylindrical nanoresonators, which would manifest in the reflectance of arrays comprised of such nanoresonators ${ }^{6,35}$. Sub-nanometer phonon oscillations could also be visualised explicitly with electron energyloss spectroscopy ${ }^{38,39}$. These nonlocal effects will be of practical relevance for a number of current nanophoton- 
ics investigations, including the attempt to reach singlemolecule vibrational strong coupling or few-electrons strong coupling ${ }^{40,41}$.

\section{ACKNOWLEDGEMENTS}

S.D.L. is supported by a Royal Society Research Fellowship and the Philip Leverhulme Prize of the Leverhulme Trust. The authors acknowledge support from the Royal Society Grant No. RGF $\backslash \mathrm{EA} \backslash 181001$.
* Corresponding author: s.de-liberato@soton.ac.uk

1 J. Khurgin, Nanophotonics 7, 2305 (2017).

2 J.-J. Greffet, R. Carminati, K. Joulain, J.-P. Mulet, S. Mainguy, and Y. Chen, Nature 416, 61 (2002).

3 R. Hillenbrand, T. Taubner, and F. Keilmann, Nature 418, 159 (2002).

4 J. D. Caldwell, L. Lindsay, V. Giannini, I. Vurgaftman, T. L. Reinecke, S. A. Maier, and O. J. Glembocki, Nanophotonics 4, 44 (2015).

${ }^{5}$ B. T. Spann, R. Compton, D. Ratchford, J. P. Long, A. D. Dunkelberger, P. B. Klein, A. J. Giles, J. D. Caldwell, and J. C. Owrutsky, Physical Review B 93, 085205 (2016).

6 C. R. Gubbin, F. Martini, A. Politi, S. A. Maier, and S. De Liberato, Physical review letters 116, 246402 (2016).

7 N. C. Passler, C. R. Gubbin, T. G. Folland, I. Razdolski, D. S. Katzer, D. F. Storm, M. Wolf, S. De Liberato, J. D. Caldwell, and A. Paarmann, Nano letters 18, 4285 (2018).

8 C. T. Ellis, J. G. Tischler, O. J. Glembocki, F. J. Bezares, A. J. Giles, R. Kasica, L. Shirey, J. C. Owrutsky, D. N. Chigrin, and J. D. Caldwell, Scientific Reports 6, 32959 (2016).

9 C. R. Gubbin, S. A. Maier, and S. De Liberato, Physical Review B 95, 035313 (2017).

10 A. M. Dubrovkin, B. Qiang, T. Salim, D. Nam, N. I. Zheludev, and Q. J. Wang, Nature Communications 11, 1863 (2020).

11 C. R. Gubbin and S. De Liberato, ACS Photonics 4, 1381 (2017).

12 I. Razdolski, N. C. Passler, C. R. Gubbin, C. J. Winta, R. Cernansky, F. Martini, A. Politi, S. A. Maier, M. Wolf, A. Paarmann, and S. De Liberato, Physical Review B 98, 125425 (2018).

13 P. Li, X. Yang, T. W. W. Maß, J. Hanss, M. Lewin, A.K. U. Michel, M. Wuttig, and T. Taubner, Nature Materials 15, $870 \mathrm{EP}$ (2016).

14 P. Li, I. Dolado, F. J. Alfaro-Mozaz, F. Casanova, L. E. Hueso, S. Liu, J. H. Edgar, A. Y. Nikitin, S. VÃ@lez, and R. Hillenbrand, Science 359, 892 (2018).

15 K. Chaudhary, M. Tamagnone, X. Yin, C. M. Spägele, S. L. Oscurato, J. Li, C. Persch, R. Li, N. A. Rubin, L. A. Jauregui, K. Watanabe, T. Taniguchi, P. Kim, M. Wuttig, J. H. Edgar, A. Ambrosio, and F. Capasso, Nature Communications 10, 4487 (2019).

16 R. Berte, C. R. Gubbin, V. D. Wheeler, A. J. Giles, V. Giannini, S. A. Maier, S. De Liberato, and J. D. Caldwell, ACS Photonics 5, 2807 (2018).

17 C. Ciracì, J. B. Pendry, and D. R. Smith, ChemPhysChem 14, 1109 (2013).

18 N. A. Mortensen, S. Raza, M. Wubs, T. Søndergaard, and S. I. Bozhevolnyi, Nature Communications 5, 3809 (2014).

19 C. Ciracì, R. Hill, J. Mock, Y. Urzhumov, A. FernándezDomínguez, S. Maier, J. Pendry, A. Chilkoti, and D. Smith, Science 337, 1072 (2012).
20 D. C. Ratchford, C. J. Winta, I. Chatzakis, C. T. Ellis, N. C. Passler, J. Winterstein, P. Dev, I. Razdolski, J. R. Matson, J. R. Nolen, J. G. Tischler, I. Vurgaftman, M. B. Katz, N. Nepal, M. T. Hardy, J. A. Hachtel, J.-C. Idrobo, T. L. Reinecke, A. J. Giles, D. S. Katzer, N. D. Bassim, R. M. Stroud, M. Wolf, A. Paarmann, and J. D. Caldwell, ACS Nano 13, 6730 (2019).

21 O. S. Ojambati, R. Chikkaraddy, W. D. Deacon, M. Horton, D. Kos, V. A. Turek, U. F. Keyser, and J. J. Baumberg, Nature Communications 10, 1049 (2019).

22 F. Neubrech, C. Huck, K. Weber, A. Pucci, and H. Giessen, Chemical Reviews 117, 5110 (2017).

23 R. O. Jones, Rev. Mod. Phys. 87, 897 (2015).

24 N. Rivera, J. Coulter, T. Christensen, and P. Narang, (2018), arXiv:1809.00058.

25 C. R. Gubbin and S. De Liberato, Phys. Rev. X 10, 021027 (2020)

26 "The model for spherical nanoparticles is available to download here:," https://github.com/SDeLiberato/ Nonlocal_FEM.

27 C. Trallero-Giner and F. Comas, Philosophical Magazine B 70, 583 (1994).

28 D. F. Nelson, Phys. Rev. Lett. 76, 4713 (1996).

29 P. R. Rimbey and G. D. Mahan, Solid State Communications 15, 35 (1974).

30 S. I. Pekar, Journal of Physics and Chemistry of Solids 5, 11 (1958).

31 B. K. Ridley, in Quantum Well and Superlattice Physics $I V$, Vol. 1675 (International Society for Optics and Photonics, 1992) pp. 492-498.

32 S. Raza, G. Toscano, A.-P. Jauho, M. Wubs, and N. A. Mortensen, Physical Review B 84, 121412 (2011).

33 K. Karch, P. Pavone, W. Windl, O. Schütt, and D. Strauch, Phys. Rev. B 50, 17054 (1994).

34 M. L. Cohen and S. G. Louie, Fundamentals of Condensed Matter Physics (Cambridge University Press, 2016).

35 J. D. Caldwell, O. J. Glembocki, Y. Francescato, N. Sharac, V. Giannini, F. J. Bezares, J. P. Long, J. C. Owrutsky, I. Vurgaftman, J. G. Tischler, et al., Nano letters 13, 3690 (2013).

36 A. Fernández-Domínguez, A. Wiener, F. García-Vidal, S. Maier, and J. Pendry, Physical review letters 108, 106802 (2012).

37 E. Prodan, C. Radloff, N. J. Halas, and P. Nordlander, Science 302, 419 (2003).

38 S. Raza, N. Stenger, A. Pors, T. Holmgaard, S. Kadkhodazadeh, J. B. Wagner, K. Pedersen, M. Wubs, S. I. Bozhevolnyi, and N. A. Mortensen, Nature Communications 5, 4125 (2014).

39 M. J. Lagos, A. Trügler, U. Hohenester, and P. E. Batson, Nature Communications 543, 529 (2017).

40 J. Keller, G. Scalari, S. Cibella, C. Maissen, F. Appugliese, E. Giovine, R. Leoni, M. Beck, and J. Faist, Nano Lett. 
17, 7410 (2017).

41 D. Ballarini and S. De Liberato, Nanophotonics 8, 641 (2019). 